\title{
Digitale Zusammenarbeit
}

\author{
Susanne Robra-Bissantz $\cdot$ Dominik Siemon
}

(C) Springer Fachmedien Wiesbaden GmbH, ein Teil von Springer Nature 2019

Kontrolle ist gut, Vertrauen ist besser. In Organisationen und Unternehmen arbeiten Menschen zusammen, eine Chefin verteilt Aufgaben und kontrolliert deren Erfüllung. So einfach funktioniert Zusammenarbeit und noch heute erscheint sie vielen eigentlich nicht weiter erklärungsbedürftig. Wieder einmal ist es die zunehmende Digitalisierung, die das Leben schwerer macht. Auf der negativen Seite macht die in digitalen Netzen häufig unübersichtliche und sozusagen unsichtbare Aufgabenverteilung und -erledigung eine umfassende Kontrolle fast unmöglich. Auf der positiven Seite jedoch ermöglichen und fördern heutige digitale Netze auch eine Kooperation, die derjenigen in kopräsenten Settings sehr ähnlich ist: die Kooperationspartner sind präsent und erkennbar, Kommunikation kann Emotionen transportieren und soziale Beziehungen entstehen.

Das vorliegende Heft der HMD - Praxis der Wirtschaftsinformatik ist daher der digitalen Zusammenarbeit in vielfältigen Facetten gewidmet. Denn die heutige Forschung und Praxis der Zusammenarbeit erkennen zunehmend, dass gegenseitiges Vertrauen, gleiche Ziele oder passende Anreize in vielen Szenarien unerlässlich für den Erfolg einer Kooperation sind und dabei teilweise so stark wirken, dass Kontrolle in den Hintergrund rückt.

Der ebenso interessante wie angenehm lesbare Artikel von Dietrich von der Oelsnitz „It don't come easy: Erfolgsvoraussetzungen von Kooperationen“ führt in die aktuelle Ausgabe unserer Zeitschrift für Wirtschaftsinformatik mit einem Blick auf die Erfolgsfaktoren kooperativen Wirtschaftens ohne spezifische Berücksichtigung von digital unterstützten Informationssystemen ein. Er zeigt an vielfältigen Beispielen auf, dass Kooperation nur mit einem strategischen Blick aller Partner, der

S. Robra-Bissantz $\cdot$ D. Siemon $(\bowtie)$

Institut für Wirtschaftsinformatik, Lehrstuhl für Informationsmanagement,

Technische Universität Braunschweig, Braunschweig, Deutschland

E-Mail: d.siemon@tu-braunschweig.de 
mehr als kurzfristige Erfolge sieht, gelingt. Fachlich ähnliche Fähigkeiten sowie ein ähnliches soziales Milieu (geringe kognitive und relationale Distanz) gehören dabei ebenso zum Erfolg wie ein Teamfokus der Partner, der durch aktive Führung eine Art Netzwerkidentität bewirkt. Darüber hinaus tritt Kooperationskompetenz auf individueller und organisationaler Ebene, die beispielsweise dazu führt, dass Kooperation nicht einfach von selbst laufen gelassen, sondern vielmehr gesteuert wird und dabei die Partner über organisationales Lernen stärkt.

Der Grundlagenartikel „Kooperationen in der digitalen Wirtschaft“" schlägt eine Strukturierung unterschiedlicher Formen der Zusammenarbeit vor und zeigt darauf aufbauend so genannte Mechanismen der Kooperation auf, die das gemeinsame Arbeiten befördern. Dabei reicht die Spannweite von der stark hierarchisch geprägten Zusammenarbeit in Unternehmen über Teams, die zusammengestellt und geführt werden, bis hin zu einer freiwilligen und auf verschiedenen Anreizen basierenden Kooperation, wie man sie beispielsweise in sozialen Medien (sozialen Netzen, sozialer Software etc.) findet. In allen Formen ist es notwendig, dass die Partner sich derart zusammenfinden, dass sie gleiche Ziele haben - dabei können dies sowohl eher kurzfristige Sachziele oder aber eher langfristige Beziehungsziele sein. Partner treten dann Kooperationen manchmal freiwillig bei - wenn gleiche Ziele vor der Gruppenbildung verabredet sind. Sie sind damit - ebenso wie potenziell im weiteren Verlauf der Kooperation - indirekt koordiniert, denn kein Mechanismus gibt eine Reihenfolge oder bestimmte Abläufe vor. Ebenso ist eine direkte Koordination, in der die Software die Rolle eines Moderators übernimmt, möglich - bekannt ist diese aus dem im amerikanischen Raum beforschten Collaboration Engineering. Die Differenzierung in verschiedene Kooperationsformen führte, zunächst in der englischen Sprache, aber heute auch im Deutschen, zu einer Unterform der Kooperation, die in der Forschung als Kollaboration bezeichnet wird. Sie bezeichnet das gemeinsame Arbeiten an einem ,Werkstück“, wie sie besonders in der digitalen Zusammenarbeit häufig (z.B. in einem Dokument oder auf einer Plattform) zu finden ist. Ein besonderer Schwerpunkt des Beitrags ist die digitale soziale Kollaboration, auf einer digitalen Plattform und mit indirekter Koordination. Hier zeigen sozialwissenschaftliche Theorien auf, wie eine digitale Plattform über ihre Gestaltung Anreize für die freiwillige Mitwirkung eines Partners ebenso wie für sein kooperatives Verhalten setzen kann. Dies gelingt beispielsweise, wenn die Plattform gemeinsame Ziele über ein gemeinsames mentales Modell (Shared Mental Model) befördert oder die Angleichung von Zielen einzelner Partner an das Gruppenziel (Yield Shift Theory) bewirken kann. Kooperatives Verhalten bedingt beispielsweise Vertrauen ebenso wie Reziprozität. Group Awareness (das gegenseitige Kennen und Schätzen der Partner), Team Emotional Intelligence (die Wahrnehmung und der gemeinsame Umgang mit Emotionen der Partner) oder eine Transparenz über Beiträge aller Partner (Evaluation Apprehension) können dies unterstützen.

Zwei weitere Artikel beschäftigen sich eher grundlegend mit digitaler Kooperation.

Bittner et al. greifen in ihrem Artikel zur Mensch-Maschine-Kollaboration die Tradition des direkt über ein digitales System koordinierten Collaboration Engineering auf. Anhand von drei Szenarien werden hierzu jedoch die Besonderheiten herausgearbeitet, wenn einer der Partner nicht menschlich, sondern IT-basiert und 
mit künstlicher Intelligenz versehen ist. Die Autoren zeigen auf, dass dann neue Herausforderungen in der Formulierung gemeinsamer Ziele, in der Verteilung von Aufgaben auf die Partner, in der Verhaltensmodellierung und ggf. Kennzeichnung des digitalen Partners sowie insgesamt im grundsätzlichen Prinzip der IT-basierten Moderation bestehen.

In Benke und Maedches Artikel „Die Rolle von Affekt und Kognition in Kollaborationswerkzeugen bei der Gestaltung und Nutzung von Kollaborationswerkzeugen“" steht als indirekter Mechanismus die konkrete Situation eines Kooperationspartners im Vordergrund des Interesses. Eine Literaturstudie zeigt hierzu die Vielfalt veröffentlichter Studien auf und zeigt anhand einiger (bestehender) Konzepte und Technologien, wie es in einem digitalen Umfeld gelingt, beispielsweise Emotionen oder Aufmerksamkeit zu erkennen, zu verarbeiten und den Kooperationspartnern zur Verfügung zu stellen.

Kooperation fördert gemeinsames Wissen. Es ist besser, Wissen zu teilen, gemeinsam zu nutzen und auch gemeinsam zu erwerben.

Insgesamt vier Artikel beschäftigen sich mit Kooperation in Wissenserwerb und Wissensaustausch. Dabei werden in zwei Artikeln Spielmechanismen eingesetzt ein im Rahmen der Kooperation weit verbreiteter und erfolgreicher Anreiz zur Mitwirkung, der allerdings im folgenden Prozess gewöhnlich eher Konkurrenz als kooperatives Verhalten fördert.

Friedrich et al. bringen in ihrem Artikel „Quizzen für den Unternehmenserfolg: Weiterbildung mittels Enterprise Social Networks neu gestalten“ ein typisches Spiel - eine bereits erfolgreiche Implementierung eines Quiz - zur Weiterbildung von Mitarbeitern in ein Enterprise Social Network. Auf den Ebenen Mensch, Technik und Organisation werden Rahmenbedingungen für eine nachhaltige erfolgreiche Einführung aufgezeigt.

Eckardt und Finster führen in ihrem Artikel „Kollaboration oder Wettbewerb: ein Vergleich der Motivation beim Game-based Learning“ ein Experiment durch, in dem ein Game-based Learning einmal gemeinsam und mit Wettbewerbselementen und einmal allein kollaborativ mit dem Fokus auf dem Erreichen eines gemeinsamen Ziels gespielt wird. Es zeigt sich, dass die Motivation der Lernenden allein über Kollaboration ebenso hoch ist, wie im Szenario mit Konkurrenz und Kooperation - wobei sich die Lernenden zudem einig sind, dass das kollaborative Lernen ihnen Spaß macht.

Schwieriger als kooperatives Lernen ist der Austausch von Wissen, vor allem in organisatorischen Settings. Wilms et al. analysieren in ihrem Artikel ,Wissensaustausch in Unternehmen: Wahrnehmung von Enterprise Social Software als Tool für den Austausch von sicherheitsrelevantem Wissen“ empirisch eine häufig genannte, wesentliche Barriere des Wissensaustauschs: sicherheitsrelevantes Wissen. Es zeigt sich, dass MitarbeiterInnen, die über bessere Kenntnisse hinsichtlich der Berechtigungsstrukturen verfügen, deutlich stärker bereit sind, derartiges Wissen zu teilen.

Es bleibt, wie Bitzer und Werther in ihrem Artikel „Herausforderungen und Lösungsansätze durch den Einsatz von digitalen Zusammenarbeitssystemen im Wissensmanagement in einem globalen Mehrmarken-Konzern" konstatieren, dass vielfältige Rahmenbedingungen, kultureller, technischer und organisatorischer Art bedacht werden müssen. Die Autoren zeigen auf, wie diese in der Praxis adressiert 
werden und weisen, untermauert mit Beispielen, vor allem darauf hin, dass eine deutliche Differenzierung von Aufgabenbereichen verschiedener sozialer Systeme oder aber eine Integration zusammengehöriger Funktionalitäten einen wesentlichen ersten Schritt zum Erfolg darstellt.

In Praxis und Forschung der betrieblichen Kooperation spielen ihre Potenziale in der gemeinsamen Innovation eine zumindest ebenso große Rolle, wie im gemeinsamen Umgang mit Wissen. Dies wird besonders deutlich, wenn man sich klarmacht, dass Kreativität immer auf dem Zusammenspiel von unterschiedlichen Neigungen und Kompetenzen beruht: divergentes Denken entwickelt konvergentes Denken weiter, Risikofreude ergänzt Risikoaversität und Sinnhaftigkeit kommt mit Neuartigkeit zusammen.

Insgesamt vier Artikel ranken sich in diesem Heft um kreative Prozesse im Design Thinking.

Redlich et al. starten mit den speziellen Anforderungen eines KMUs mit industrienahen Dienstleistungen und passen sowohl Design Thinking Phasen, Methoden und Tools daran an. Als übergeordnete Erkenntnis verbleibt, dass das häufig in realen Settings einfacher zu erreichende Mindset, wie beispielsweise Empathie, Teamgeist und Offenheit der unterschiedlichen Mitwirkenden für neue Lösungen ausschlaggebend für den Erfolg der Innovationsentwicklung ist.

Lembcke et al. gehen am Beispiel des Hasso-Plattner-Instituts von Situationen aus, die sich bei der Kollaboration im Design Thinking als besonders herausfordernd darstellen und identifizieren, ähnlich wie im Artikel von Redlich et al., insbesondere die emotionalen (weichen) Teamfaktoren - wie gegenseitiges Vertrauen und der konstruktive Umgang mit unterschiedlichen Meinungen, Kompetenzen und Erfahrungen - als erfolgsentscheidend. Sie zeigen anschließend beispielhaft auf, wie dementsprechend Informationssysteme zur Teamunterstützung gestaltet werden können.

Mirbabaie et al. nutzen die auch bei Bittner et al. grundlegende Kollaboration mit dem digitalen Partner als Moderator in einem erweiterten Brainstorming: in diesem Fall mit einem auf künstlicher Intelligenz basierenden Chat Bot. Dieser ist basierend auf eine Reihe von Erkenntnissen zur Teamarbeit gestaltet und nutzt insbesondere die auch im Umgang mit Wissen anerkannten Spielmechaniken - in diesem Fall zur Motivation um beispielsweise soziales Faulenzen zu vermeiden.

Hofer et al. sehen als potenzielle Aufgabe eines digitalen Tools im Design Thinking die Dokumentation des Prozesses sowie der Ergebnisse. Basierend auf umfassenden Anforderungen wurde im Artikel ,Ich weiß was ihr letzte Sitzung getan habt - Entwicklung und Anwendung eines Softwarewerkzeuges zur Dokumentation von Design Thinking-Projekten“ ein Prototyp entwickelt. Dieser bereichert den Prozess des Design Thinkings durch eine systematische Dokumentation, aber auch durch die Bereitstellung der in Kreativität notwendigen Wissensbasis, sowie die wiederum moderierte Ermöglichung von parallelem Arbeiten.

Zwei Praxisbeispiele im speziellen Umfeld der Beteiligung von Bürgern runden die Beiträge zur Kooperation in der Innovation ab.

Billert und Peters sehen in ihrem Artikel „Die Digitalisierungsstraße für die Stadt der Zukunft - Kollaborative Entwicklung eines Portals für bürger-initiierte Dienstleistungsentwicklung im Kontext einer Smart City“ Kooperation als Basis städtischer Dienstleistungen, die sich sowohl in der Entwicklung des Bürgerportals, in der Ide- 
enfindung für neue Dienstleistungen sowie in städtischen Dienstleistungen an sich finden. Basierend auf den Grundlagen des Service und Collaboration Engineering sowie des Design Science und der Bürgerbeteiligung entsteht das Portal unter Partizipation der Interessensgruppen in zwei Evaluationsrunden und Iterationen.

Im Artikel „Open Innovation in der Politik: Internetgestützte Zusammenarbeit in politischen Parteien am Beispiel der ÖVP in Österreich“"von Schmidthuber et al. wird kein Team, sondern eine gesamte Partei mit freiwilligen Akteuren zur Mitwirkung am neuen Parteiprogramm aufgefordert. Sehr ähnlich bleiben - laut der empirischen Studie - die Anforderungen an Offenheit, kopräsente und digitale Formate, ein Zusammenführen der Community und das innovative Mindset. Neu und relevant insbesondere für freiwillige Kooperationen im politischen Umfeld ist die Identifikation eines so genannten Participation Gap, da einige Bürgergruppen stärker als andere digital partizipieren können und wollen.

Nicht nur bei Bürgern und Parteien und nicht nur in der Innovation findet auch organisationsübergreifende Kooperation statt - eine vermutete Zukunft liegt auch darin, mit und für Kunden kooperative Settings statt der typischen marktlichen Koordination anzustreben. Dies zeigen die folgenden vier Beiträge.

Herrenkind et al. betrachten die „Kollaborative Wertschöpfung im Carsharing“. Sie identifizieren hier insgesamt 5 kollaborative Wertschöpfungsmuster mit unterschiedlichen Partnern und Anreizen, wie beispielsweise die Reinigung der Automobile durch Kunden oder deren Bereitstellung an stark frequentierten Orten. Anhand einer Literaturanalyse können sie diese auf eine Reihe von weiteren Sharing Economies übertragen und gewinnen so wertvolle Erkenntnisse zu den Potenzialen einer gemeinsamen Wertschöpfung von Unternehmen und Kunden.

Auch der Beitrag „Ein Rezept für die Apotheke 2.0 - Wie Informations- und Kommunikationstechnologie die intersektorale Zusammenarbeit in der Gesundheitsversorgung stärken kann“"geht davon aus, dass durch Kooperation aller Akteure im Gesundheitssystem gemeinsame Ziele, wie Kundenzufriedenheit, Patientengesundheit, Therapietreue und Kosteneffizienz, erzielt werden können. Hierzu identifiziert der Beitrag fast 40 Best Practices der Digitalisierung für Apotheken und leitet mit Experten wesentliche Handlungsempfehlungen für Apotheken im Konkurrenzkampf mit Online-Angeboten ab. Diese umfassen im Wesentlichen eine bessere (digitale) Kooperation der anbietenden Akteure, im eRezept oder digitalen Medikationsplan, sowie mit den Kunden, mit neuen Lieferservices oder neuen Angeboten für Senioren.

Im Verhältnis zwischen Online- und Offline-Angeboten für den Handel schlagen Wichert et al. in ihrem konzeptionellen und theoretisch fundierten Beitrag „Instrumente der Interaktionsintegration im Omni-Channel Handel“" vor, ausgehend von den potenziellen Kontakten in den Kaufprozessphasen auf Integration und eine gemeinsame Steuerung der Interaktionen verschiedener Kanäle zum Kunden zu setzen und dabei sowohl die Kooperation zwischen verschiedenen Händlern und Dienstleistern als auch zwischen Händlern und Kunden zu fördern.

Neue Wege der Digitalisierung im Handel geht schließlich der Beitrag „Ich fühle mit dir! Können empathische virtuelle Assistenten den stationären Einzahlhandel unterstützen?" von Meyer und Strohmann. Die Autoren schlagen hier vor, den Händler im stationären Handel durch einen digitalen Assistenten zu unterstützen, der mithilfe von Ansätzen der emotionalen Intelligenz eine Kooperation zwischen Händler 
6

S. Robra-Bissantz, D. Siemon

und Kunde fördert. Dazu erkennt das empathische Informationssystem verschiedene emotionale Situationen des Kunden anhand seines realen und virtuellen Kontextes und bietet dem Händler dazu passende Reaktionsmöglichkeiten an. Der bestehende Prototyp ist aus Expertengesprächen im stationären Handel entstanden und soll ebenda getestet werden.

Liebe Leser, eine Fülle von interessanten Beiträgen dieser HMD lockt Sie in ganz besondere Aspekte der digitalen Zusammenarbeit, die weit über eine Vernetzung von und Datenübertragung zwischen Unternehmen hinausgeht. IT von heute und morgen nimmt in der Kooperation unterschiedliche Rollen ein: vom Moderator über die digitale Plattform mit besonderen Mechanismen bis zum Team-Mitglied. Sie wird gezielt dahingehend gestaltet, dass sie den wesentlichen weichen Anforderungen, die schon immer mit einer Kooperation zwischen Menschen einhergehen, gerecht werden kann und führt, auch aus diesem Grund, kopräsente und digitale Kooperationsräume im Sinne einer postdigitalen Unterstützung von Menschen zusammen. Wir wünschen Ihnen viel Freude beim Entdecken neuer Erkenntnisse.

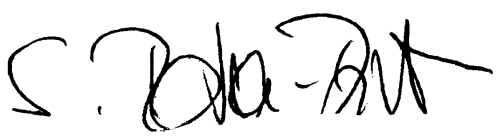

Susanne Robra-Bissantz

D. Simon

Dominik Siemon

Springer 